\title{
Hitless Wavelength Assignment in Filterless Optical Access Networks
}

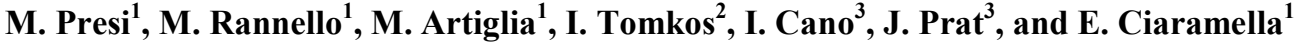 \\ ${ }^{1}$ TeCIP Institute, Scuola Superiore Sant'Anna, via G. Moruzzi, 1 - 56124 - Pisa (ITALY) \\ ${ }^{2}$ Athens Information Technology, Marousi 15125 , Greece \\ ${ }^{2}$ Universitat Politecnica de Catalunya (UPC), c. Jordi Girona 1-3, 08034 Barcelona, Spain \\ Tel: +39050 549 2269, e-mail: marco.presi@sssup.it
}

\begin{abstract}
Advantages offered by coherent detection systems in access networks are not limited to the increase of power budget due to the superior sensitivity. Channel selectivity is another intriguing feature offered by coherent systems that can be exploited to enable advanced system and network functionalities for access systems. Among them, we focus here on the implementation of filterless optical networks and network reconfiguration capabilities which might be required to satisfy dynamic load balancing requests and new terminal activations.

We show that in the access domain these functionalities do not require DSP-aided coherent receivers, but can be easily realized by means of simpler, fully analogue real-time coherent terminals matching the access network low-cost paradigm. This paper discusses these concepts and how they can be experimentally implemented by using a novel wavelength allocation algorithm and real-time analogue coherent transceivers based on DFB lasers, whose wavelengths are tuned by a simple temperature control.
\end{abstract}

Keywords: optics communications, coherent detection, analogue processing, filterless access networks.

\section{INTRODUCTION}

The advent of coherent receivers [1] has been a key enabler for systems of increased performance in terms of spectral efficiency, transmission impairments mitigation, adaptive signalling rates and, more in general a key building block of the future Software Defined Networks (SDN) [2]. These concepts have been very well received and implemented in the "core" segments of the Network. Yet there is still a Network segment where the adoption of coherent systems is still under debate: the Access Network. This might be surprising, since the Access Network may benefit by the adoption of coherent detection technologies for many reasons. As an example, coherent detection offers receivers if improved sensitivity, which allow for networks of extended power budget, and enable the $\lambda$-to-the-user functionality which ensure scalability, unshared bandwidth, grid-less operations and infrastructure compatibility with legacy filterless optical networks [3].

A typical system that realize this functionality is depicted in Fig. 1: each Optical Networking Unit (ONU) is reached by a dedicated wavelength, and no optical filters acting as WDM demultiplexers are implemented across the network. In this case a WDM MUX might be still used at the Optical Line Terminal (OLT) to mitigate mux losses when a large number of wavelength should be provided to the network [3]. This scheme requires also control and monitoring systems that keep and manage a map of active channels and the relative ONU association.

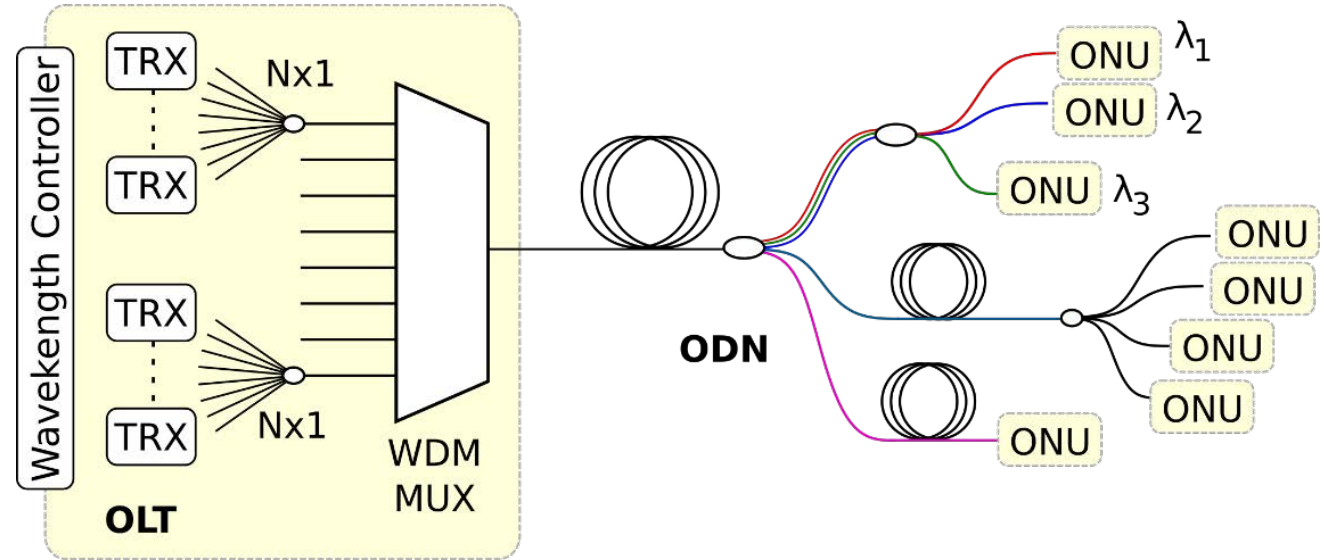

Figure 1. General scheme of a $\lambda$-to-the-user access network whose optical distribution network is filterless.

Reasons behind hesitations in adopting coherent transceivers are related to two main factors: the first is the rapid evolution of edge network services market which does not allow easy forecasts on access system requirements; the second is that historically in the access domain technical choices have been always dictated by "cost" rather than "performance" issues. 
This latter consideration suggests that if coherent technologies will be ever considered for adoption in the Access domain such adoption will not consist in a simple "as-is" use of core-network transceivers; the adoption might be favoured only after the introduction of transceivers of reduced complexity providing the required functionalities and performance level at an affordable cost.

This constraint has driven a huge R\&D effort to develop coherent receivers suitable for access networks, featuring dedicated Digital Signal Processing (DSP) units [4] and integrated optical front-ends [5]. A different strategy has been to investigate a totally different class of coherent transceivers based on low-cost hardware and analogue signal processing, aiming not only at a cost reduction, but also at an increased energy efficiency [6].

Now that low-cost coherent transceivers have been developed and tested in UDWDM filterless scenarios, it is important to address critical operating issues. ONU activation, for example, is one of those issues, because when new $\lambda \mathrm{s}$ are added to the network it is important to preserve the status of already running links (i.e. the aforementioned operation must occur without introducing interference or causing service disruption on other active channels). Similarly, when the network requires a reconfiguration which implies a $\lambda$ tuning of operating channels, such a reconfiguration must occur without service interruption on all the interested channels.

In this contribution we address this specific problems and illustrate a recently proposed wavelength management algorithm which allows both wavelengths activation and reconfiguration in a fully hitless way, i.e. without introducing impairments on activating and already active channels [7]. The proposed algorithm is quite general and it is not dependent on a specific transceiver implementation: although conceived to work in coherent detection systems (either analogue or DSP based), it might be also adopted in filterless systems based on directdetection. However, it addresses specific problems of access networks. Indeed, it requires only a monotonic laser wavelength control, in favour of simplified hardware and control systems, as demanded by Access Network applications.

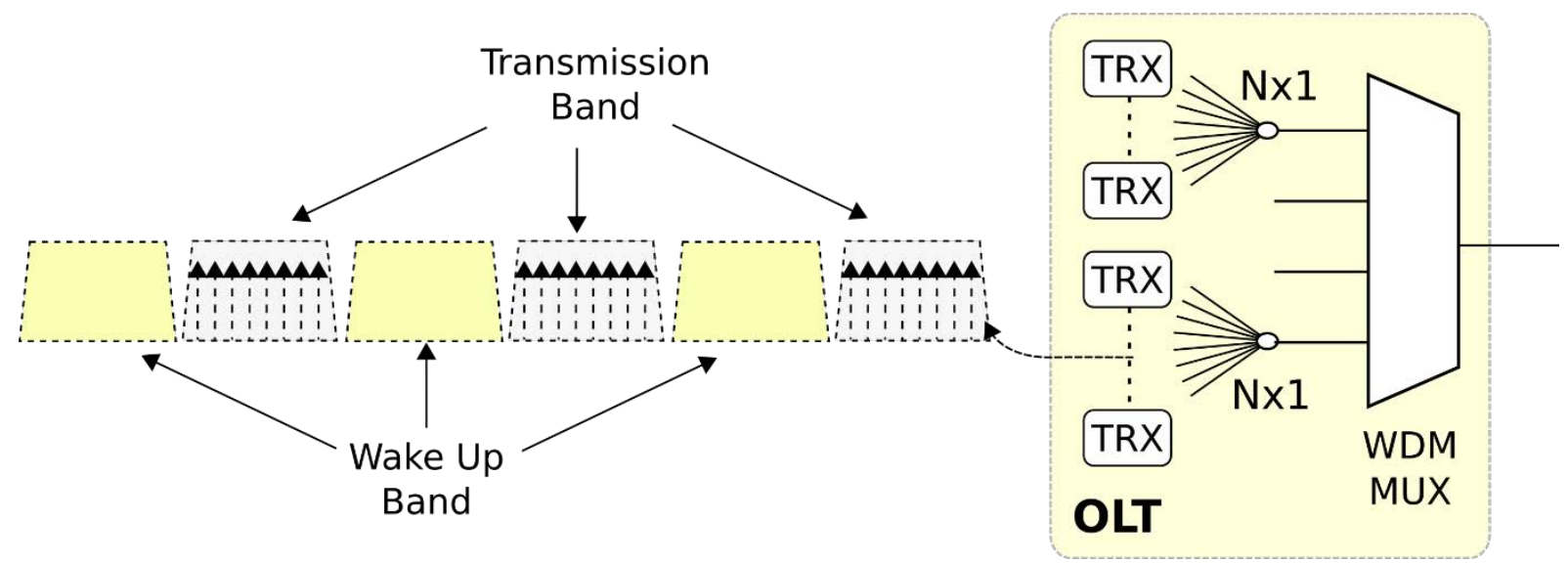

Figure 2. When hybrid power splitting and wavelength (de)multiplexing is implemented, wake-up zones are naturally available in the frequency spectrum.

\section{HITLESS WAVELENGTH ACTIVATION AND RECONFIGURATION}

The first issue that we analyse here is the ONU activation. When a laser is turned on, it is subject to strong frequency drifts before it reaches a steady-state emission at a fixed wavelength. Such frequency drifts might be extremely large, up to tens of $\mathrm{GHz}$ has been recently measured [8]. This is clearly an issue, especially when UDWM operations on 6.25 or $3.125 \mathrm{GHz}$ grids are targeted [5,6]: in this case a "turning-on" laser might be wandering over tens of running channels, clearly creating service interruptions. This problem is particularly serious in the upstream direction, since when an ONU is activated, it has no knowledge of the network status (i.e., which channels are active): at the OLT side this issue might have a minor impact, since the OLT wavelength controller has a clear knowledge of the network status. To avoid issues related to laser drifts, we introduce dedicated wavelength bands, defined as Wake-Up Zones which are frequency interleaved to the transmission bands (see Fig. 1-left). Such wake-up zones are directly available when an hybrid splitter-AWG channel multiplexing strategy is adopted at the OLT. In this case, each AWG transmission band accommodate the transmitted channels, while the unoccupied frequency region can be used as wake-up zones (see Fig. 2). As an example, an $8 \times 1.25 \mathrm{~Gb} / \mathrm{s}$ on a $6.25 \mathrm{GHz}$ grid fits nicely an AWG with a $100 \mathrm{GHz}$ period and $50 \mathrm{GHz}$ channel bandwidth: in this case, the wake-up zones are naturally interleaved to the transmission bands (with $100 \mathrm{GHz}$ spacing and $50 \mathrm{GHz}$ bandwidth). We note that the wake-up zones must be wide enough to fully contain any laser's wavelength drift associated to the activation: in the proposed example, a bandwidth of $50 \mathrm{GHz}$ is more than enough to fully contain all laser's drift [8]. 




Once that the laser has been activated in a Wake-up Zone, the negotiation can begin and the ONU sends a request to the OLT asking for a free wavelength slot: we note that a conflict might occur at this stage, in the event that more than one ONU activate simultaneously within the same Wake-up zone during the negotiation stage; however, this cannot introduce any service disruption on already active channels, while it might cause a delay on the overall activation time. The proposed algorithm specifies that the ONU is then assigned to the first highest frequency (rightmost) available channel (see Fig. 3-a). At this point, the ONU starts tuning its upstream transmitter to the allocated channel in the grid. Next channels activation occurs in the same way: the $n^{\text {th }}$ activated ONU will be allocated to the first available channel at the left (the $n^{\text {th }}$ in the grid, see Fig. 3-b and 3-c). In this way, each activated ONU never "crosses-over" other running channels and therefore no service interruption is generated, as long as the frequency translation is adiabatic. We note that the proposed activation algorithm require tuning only in one direction: in the aforementioned example, the laser wavelength is increased. This is usually achieved either by "heating" the laser or increasing its bias current. Heating the laser might be the best option when it is important to keep an eye on power consumption. Indeed, in many operational cases, it actually means to "reduce" the cooling action of the temperature control system, or in other words to reduce its power consumption. Another important point is that a monotone temperature controller is clearly simpler to design and is another mean to help towards the cost reduction. It should be noted that the activation process and the consequent beginning of real data transmission is not necessarily concluded after that the laser has finished the tuning process. Indeed, if the transceivers are capable, the communication can begin during the tuning process. This requires that while the upstream laser tunes towards the target frequency, the corresponding local oscillator at the OLT side "follows" in real-time the frequency shift. This process, is known as "push-pull". It has been first introduced by taking advantage of advanced DSP and line coding [9], but it has been also experimentally demonstrated also by using all-analogue coherent transceivers [7]. By doing this the activation time can be considerably reduced.

Another aspect that must be taken in consideration is the dynamic wavelength reconfiguration. In core networks, run-time wavelength reallocation is often requested during network operations: it satisfies dynamic load changes, light-path reconfigurations or allow for resources re-allocation. In a pure WDM Access Network scenario, such as the $\lambda$-to-the-user one, wavelength reconfigurations occur with a reduced probability, being required only when an $\mathrm{ONU}$ is shut down. In case of the proposed algorithm, the wavelength allocation process guarantees that there are no free slots on the grid, i.e. the channels on the grid are all continuously occupied (see Fig. 3-c). However, when one ONU terminates its operation, a free channel slot is left in the grid (Fig. 1-d). This slot might be filled again by a new ONU activation, but this would clearly be a non-hitless process. In order to make new activations hitless, in this case the OLT requests that all the lighted channels shift to their rightmost channel position in order to fill again the emptied slot (see Fig. 1-e and 1-f). This step is coordinated by the OLT, so that the upstream signal of each involved ONU and the respective Local Oscillators (LOs) at the upstream receivers are frequency shifted simultaneously by exploiting the "push-pull" technique. By doing so, during the frequency shift the signal-LO detuning remains within the coherent receiver bandwidth and no service interruption is generated. After this multiple reallocation, the emptied slot is moved to the rightmost position, allowing for a new hitless ONU activations. The push-pull operation is almost seamless if a digital coherent receiver is used. On the other hand in fully-analog receivers this task is more challenging since the signal-local oscillator tracking must be extremely precise during the tuning process (as rule of thumb the tolerance is about a $10 \%$ of the bit-rate). If the push-pull technique is adopted, the tuning time must not be extremely fast: even if the tuning occurs in few seconds, the activation is not affected, since the tuning channel is always operational.

Another case of interest is a new activation when a transmission band is already filled. In this case, if the laser activation occurs on the leftmost Wake-Up-Zone, as the laser shows up the wavelength controller notifies the 
ONU that the transmission band and instructs the ONU to turn on inside another Wake-Up-Zone, where the adjacent transmission band has free slots. The feasibility of the proposed algorithm depends on the tunability range of the transmitters used. DFB lasers, which are good candidates for Access Networks allows for a $300 \mathrm{GHz}$ tuning range. By considering the aforementioned scenario (multiplexing by using a cascade of $1 \times 8$ power splitters and a $100 \mathrm{GHz}$ AWG), a single laser can be allotted at least over 3 consecutive transmission bands. Of course, lasers of wider tunability offer more flexibility; yet, it has been shown that also by using random assignment of limited tenability lasers, and exploiting statistical wavelength allocation schemes, it is still feasible to realize networks supporting hundreds of users [10].

\section{CONCLUSIONS}

In conclusion we discussed a novel algorithm allowing lasers activation and frequency reconfiguration which is suitable for WDM access networks realizing $\lambda$-to-the-user systems. The algorithm considers physical constraints, i.e. tuning times and range typical of semiconductor lasers used in the Access Network domain. The proposed algorithm is not designed around a specific transceiver: it might be implemented either in direct-detection or coherent systems without loss of generality. In addition, it features a monotonic wavelength control of the involved lasers, which is particularly suitable for cost requirements typical of Access Networks.

\section{ACKNOWLEDGEMENTS}

This work has been developed within the framework of the FP7 European Project COCONUT (G.A. 318515).

\section{REFERENCES}

[1] K. Kikuchi, "Fundamentals of coherent optical fiber communications," IEEE/OSA Journal of Lightwave Technology, vol. 34, no. 1, pp. 157-179, Jan. 2016.

[2] A. Morea, O. Rival, N. Brochier, E. L. Rouzic, "Datarate adaptation for night-time energy savings in core networks," IEEE/OSA Journal of Lightwave Technology, vol. 31, no. 5, pp. 779-785, 2013.

[3] J. Prat et al., "Towards ultra-dense wavelength-to-the-user: The approach of the COCONUT project," in Proc. 15th International Conference on Transparent Optical Networks (ICTON), 2013.

[4] D. Lavery et al., "Digital coherent receivers for long-reach optical access networks," IEEE/OSA Journal of Lightwave Technology, vol. 31, no. 4, pp. 609-620, 2013.

[5] H. Rohde et al., "Coherent ultra dense WDM technology for next generation optical metro and access networks," IEEE/OSA Journal of Lightwave Technology, vol. 32, no. 10. pp. 2041-2052, 2014.

[6] J. Prat, "Technologies for a cost-effective coherent udWDM-PON," in Proc. Optical Fiber Communication Conference, 2015, paper Th3I.3.

[7] M. Presi et al., "Hitless dynamic wavelength allocation for WDM coherent PONs," in Proc. Optical Fiber Communication Conference, 2016, paper W2A.63.

[8] G. Simon et al., "Spectral and temporal analysis of the NGPON2 short-term wavelength drift for 10Gbit/s Bursts," in Proc. Optical Fiber Communication Conference, 2016, paper W4C-2.

[9] F. Cugini et al., "Push-pull defragmentation without traffic disruption in flexible grid optical networks," IEEE/OSA Journal of Lightwave Technology, vol. 31, no. 1, pp. 125-133, 2013.

[10] V. S. Zaragoza et al., "Statistical UDWDM-PONs operating with ONU lasers under limited tunability," IEEE Photonics Technology Letters, vol. 27, no. 3, pp. 257-260, 2015. 